\title{
869 LOCALLY ADMINISTERED IMMUNOTHERAPY SELF- DELIVERING RNAI PH-762 RESULTS IN ABSCOPAL CLEARANCE OF UNTREATED DISTAL TUMORS, SUGGESTING SYSTEMIC IMMUNE RESPONSE, IN A MURINE HEPATOCARCINOMA MODEL
}

Benjamin Cuiffo*, Melissa Maxwell, Dingxue Yan, Brianna Rivest, James Cardia, Simon Fricker. Phio Pharmaceuticals, Marlborough, MA, USA

Background The development of locally administered immune checkpoint inhibition (ICI) holds potential promise for enhanced activity and decreased systemic toxicity, but such an approach is challenging with the available ICI antibodies. We have previously shown that the intratumoral (IT) delivery of PH-762, a self-delivering RNAi compound targeting PD-1 based on proprietary INTASYL ${ }^{\mathrm{TM}}$ technology, can significantly inhibit tumor growth associated with changes in the immune cell population in the tumor microenvironment towards an anti-tumor phenotype. We present data showing that IT administration of PH-762 not only inhibits local tumor growth but can also elicit an abscopal effect in distal untreated tumors. The in vivo efficacy and in vitro mechanism of action support the generation of a PH-762 driven systemic anti-tumor immune response. Therefore, ICI using INTASYL is an alternative to antibody drugs for immunotherapy.

Methods To assess in vivo efficacy, Hepa1-6 cells were implanted subcutaneously into the flanks of $\mathrm{C} 57 \mathrm{BL} / 6 \mathrm{~J}$ mice. Vehicle (PBS) or murine targeting $\mathrm{PH}-762$ (mPH-762) were administered IT on Days 1, 4, 7, 10 and 14. To determine an abscopal effect cells were also implanted into the opposite flank but left untreated. Tumor volumes and body weights were recorded. In addition, in vitro mechanism of action studies were performed with CD3-stimulated human pan T cells. PD-1 mRNA knockdown was assessed by qRT-PCR; PD-1 protein expression by flow cytometry; and $\mathrm{T}$ cell function by cytokine release.

Results Treatment with IT administered mPH-762 significantly inhibited tumor growth compared with vehicle treated control tumors. Furthermore, the growth of the untreated bilateral tumor was significantly reduced with $80 \%$ of these tumors showing complete regression. Mechanism of action studies showed potent and durable silencing of PD-1. Increased release of IFN- $\gamma$, CXCL10, and IL- 6 and suppression of IL-10 release were indicators of an enhanced immune response.

Conclusions These data show that silencing PD-1 with IT administration of mPH-762 not only inhibits growth of treated tumors but elicits an abscopal effect leading to cure of distal tumors. This data and other recently published data showing evidence of a specific antitumor immune response in a tumor rechallenge model after prior treatment with INTASYL compounds, demonstrate the desired systemic immune response can be obtained with local administration of PH-762. INTASYL represent an alternative to antibody therapy for IT checkpoint blockade with potential for improved efficacy and reduced systemic toxicity which will be investigated in an upcoming clinical trial.

http://dx.doi.org/10.1136/jitc-2021-SITC2021.869 